\title{
Cardiac Resynchronization Therapy
}

\author{
State of the Art Review For the $25^{\text {th }}$ Anniversary of Cardiac Resynchronization Therapy
}

\author{
Valentina Kutyifa', 2
}

\begin{abstract}
${ }^{1}$ Clinical Cardiovascular Research Center, Cardiology Division, University of Rochester Medical Center, Rochester, NY, USA, and ${ }^{2}$ Semmelweis University, Heart Center, Budapest, Hungary
\end{abstract}

Address reprint requests to:

Valentina Kutyifa, MD, PhD, FHRS, FESC, FACC, Associate Professor of Medicine

Clinical Cardiovascular Research Center, University of Rochester Medical Center

265 Crittenden Blvd., Rochester, New York 14642-0653

E-mail: Valentina.Kutyifa@heart.rochester.edu or Valentina.Kutyifa@kardio.sote.hu

Patients with heart failure (HF) have a significant morbidity and mortality that competes with those of many cancers. In HF patients with a severely depressed left ventricular function, and a wide QRS reflecting left ventricular dyssynchrony, cardiac resynchronization therapy (CRT) has been shown to improve functional capacity, HF symptoms, and quality of life. Since the first case reports were published in 1994, there have been a large number of randomized controlled clinical trials conducted that have proven the efficacy of CRT in diverse HF populations showing a significant reduction in HF hospitalization and improved survival. CRT has, over the past 25 years, become a guideline-indicated, evidence-based device therapy for mild and advanced HF patients with severely reduced left ventricular function, and a wide QRS. Nevertheless, there are a number of factors negating beneficial response to CRT, and multiple unresolved questions to this day. This review article summarizes current available knowledge on CRT in HF patients from randomized clinical trials and other relevant studies, discusses important determinants of CRT response, and provides a selected overview of unresolved questions with future directions for research.

Keywords: Cardiac Resynchronization Therapy, Left Ventricular Dyssycnhrony, Clinical Outcomes, Clinical Trials, Future Directions

\section{Mechanism of Cardiac Resynchronization Therapy in the Failing Heart}

Patients with HF often present with an electrical conduction delay, resulting in dyssynchronous left ventricular activation, impaired left ventricular systolic function, mitral regurgitation, and a significantly reduced cardiac output (1). An electrical conduction delay is manifested either as a left bundle branch block (LBBB), right bundle branch block (RBBB), or as an intraventricular conduction delay (IVCD). Electrical activation of the ventricles in patients with $\angle B B B$ and RBBB has been previously described by Fantoni et al. (2). Patients with LBBB typically have multiple right ventricular (RV) breakthrough sites in the septum as compared to RBBB with a single RV breakthrough site. Transseptal activation time, activation time of the $R V$, and total activation time is significantly longer in RBBB as compared to $L B B B$. In patients with $\angle B B B$ or $R B B B$, there is a slow left ventricular (LV) electrical activation from the septal or anterior breakthrough sites to the apical and lateral regions. Most typically, the postero-lateral basal region is the latest activated LV area in both $L B B B$ and RBBB, providing the rationale for biventricular pacing with an LV lateral, posterolateral lead in this population. Although patients with RBBB exhibit a more advanced conduction tissue disease than LBBB that is often less amenable to CRT.

Cardiac resynchronization therapy (CRT) or biventricular pacing is delivered using a 3-lead pacing/defibrilla- 
tor system that delivers electrical stimuli to the right atrium, right ventricle, and left ventricle to synchronize the dyssynchronous LV activation in patients with conduction tissue disease and a severely reduced LV function. CRT was developed to restore the physiological atrial and ventricular contraction in the failing heart and correct atrioventricular dyssynchrony, as well as interventricular dyssynchrony and intraventricular dyssynchrony. Atrioventricular dyssynchrony, or PR-prolongation is often observed in HF patients linked to worse clinical outcomes (3). The pathophysiology of a prolonged-PR interval is primarily based on the atrial systole $(A)$ occurring early in diastole and therefore, it is superimposed on the early left ventricular filling phase $(E)$, subsequently leading to the fusion of the diastolic $E$ and $A$ waves, shortening effective diastolic LV filling time, and decreasing cardiac output. The early atrial systole uncouples the mitral valve closure from LV systole resulting in diastolic pre-systolic mitral regurgitation, decreased preload, and decreased forward stroke volume, further worsening LV function. Following CRT implantation, normalization of the PR-interval restores the physiologic $A V$-sequence, eliminating the pathologic $E$ and A fusion, and diastolic pre-systolic mitral regurgitation. Restoration of interventricular and intraventricular dyssynchrony via synchronized left ventricular (LV) and right ventricular (RV) pacing, or most often, LV pre-excitation additionally results in an immediate decrease of intra- and interventricular dyssynchrony, a decrease in mitral regurgitation, and an increase in LV contractility (4). Long-term CRT use is linked to a reduction in LV end-diastolic (LVEDV) and LV end-systolic volume (LVESV), and improvement in LV ejection fraction (LVEF), described as LV reverse remodeling $(5,6)$ (Fi-

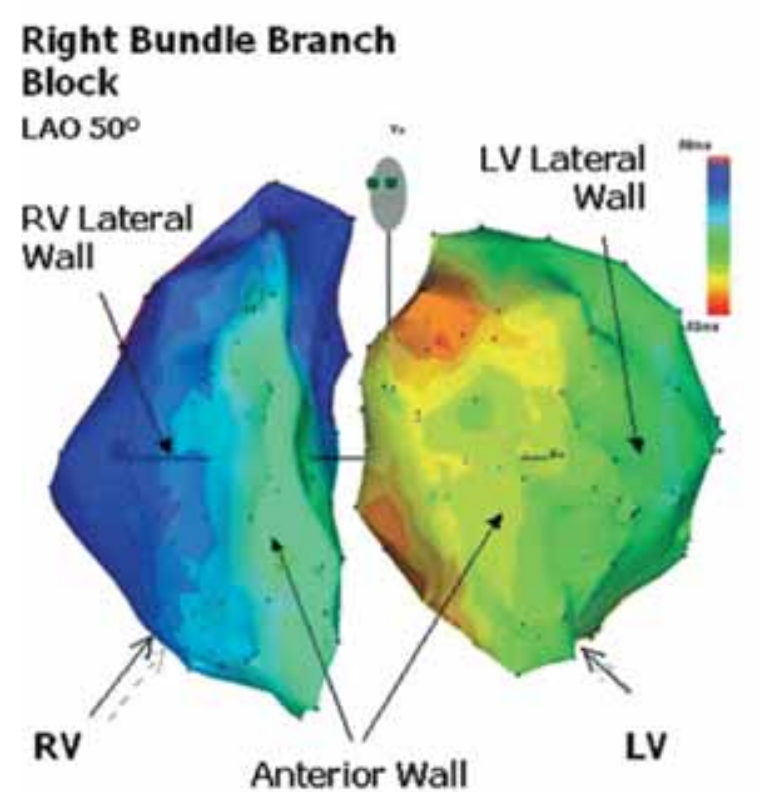

gure 1). Eliminating LV dyssynchrony, reducing LV volumes, and increasing LVEF is the hallmark of cardiac resynchronziation therapy that is associated with beneficial clinical outcomes (7) (Figure 2).

\section{Randomized Clinical Trials in Cardiac Resynchronization Therapy}

There have been a large number of randomized controlled clinical trials to ascertain the safety and efficacy of CRT or CRT-D to improve quality of life, HF symptoms, functional capacity, and clinical outcomes. These studies are summarized below in Table 1. As it has been evidenced in the early studies enrolling 50-100 patients, implantation of CRT resulted in a significant improvement in HF symptoms, functional capacity, and quality of life in HF patients with advanced HF symptoms (NYHA class III-IV), reduced LVEF $\leq 35 \%$ and a prolonged $\mathrm{QRS}$ duration (QRS $\geq 120 \mathrm{~ms}$ ) (8-10). The first double-blind randomized controlled comparison of CRT was conducted in the MIRACLE trial, enrolling 453 patients, confirming in a larger cohort of HF patients that CRT improves New York Heart Association (NYHA) class, quality of life, 6 minute walk test, and also reduces left ventricular volumes, and improves left ventricular ejection fraction, coupled with improvements in clinical symptoms.

Subsequent well-designed, randomized, controlled large clinical trials, Cardiac Resynchronization-Heart Failure (CARE-HF), and Comparison of Medical Therapy, Pacing and Defibrillation in Heart Failure (COMPANION) have shown, for the first time, that CRT also improves survival in patients with advanced HF symptoms (NYHA

\section{Left Bundle Branch Block}

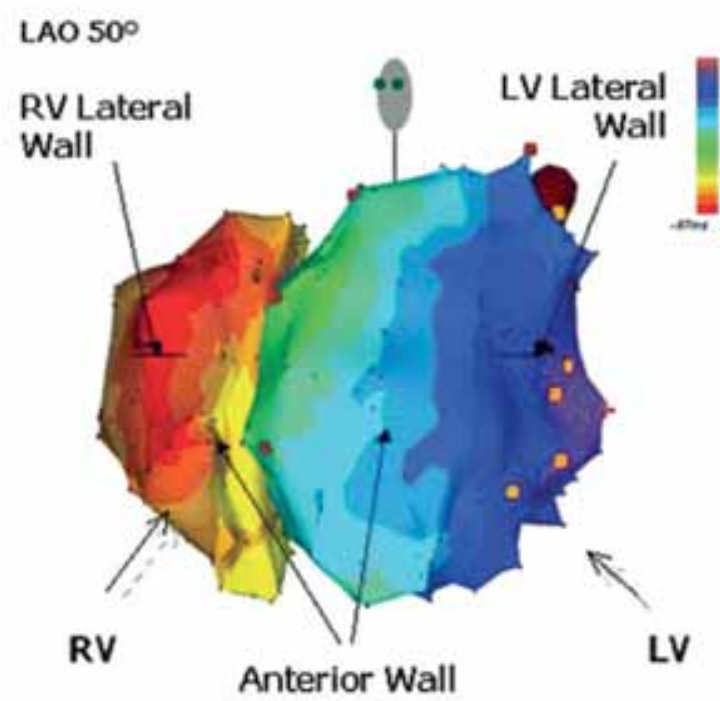

FIGURE 1. Electrical activation of the left and right ventricle in patients with right bundle branch block and left bundle branch block (2) 


\section{Baseline}

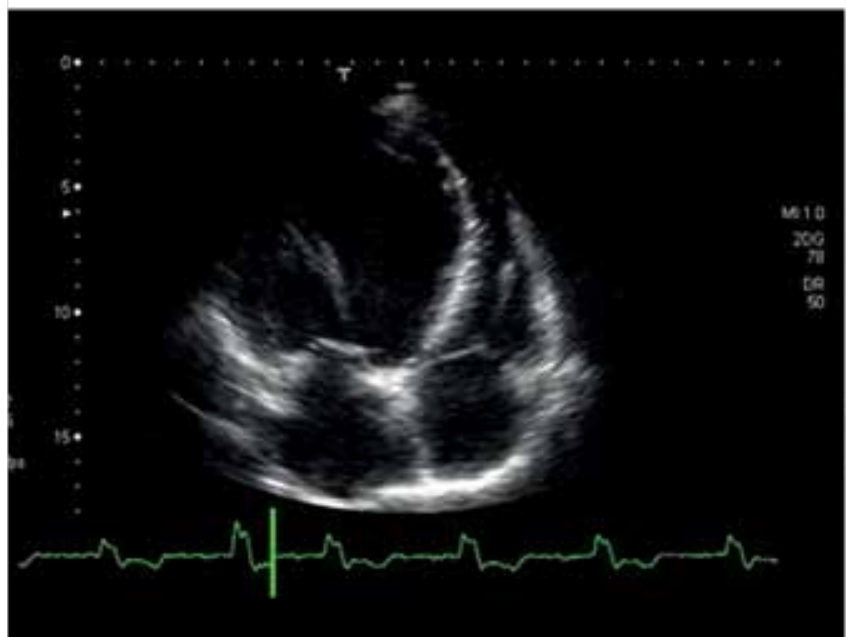

12-months after CRT

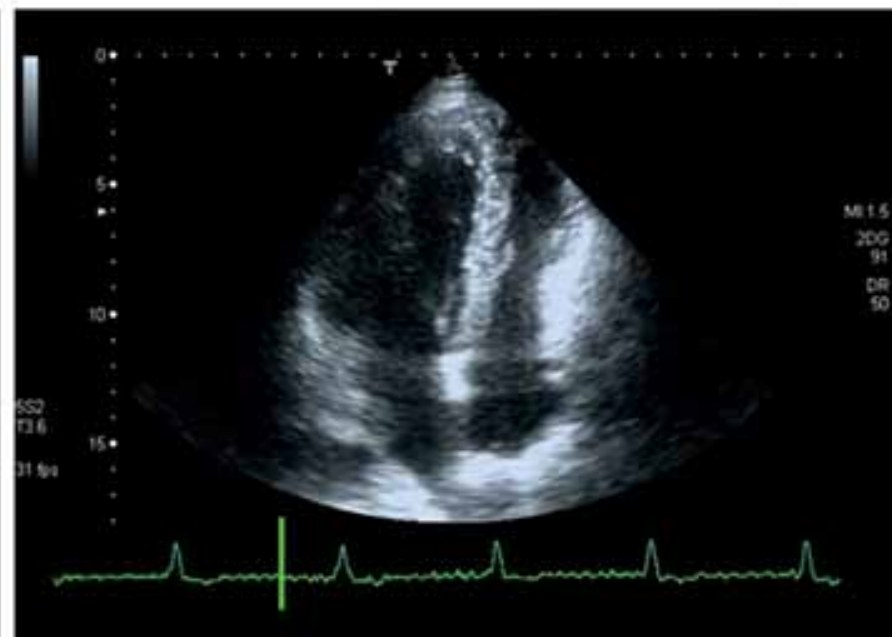

FIGURE 2. Electrical activation of the left and right ventricle in patients with right bundle branch block and left bundle branch block (2)

class III-IV), reduced LVEF $\leq 35 \%$ and a prolonged QRS duration (QRS $\geq 120 \mathrm{~ms})(9,10)$. Altogether, these studies provided the basis for current guideline-based indications for CRT in advanced HF patients with NYHA Class III or IV (11). Today, tens of thousands of advanced HF patients are implanted with CRT in Hungary, in Europe, and worldwide (12). A meta-analysis of CRT trials in advanced HF showed an overall $29 \%$ risk reduction in all-cause mortality, and a $38 \%$ risk reduction in mortality due to progressive HF (13).

Following the success of CRT in advanced HF patients, subsequent clinical trials focused on broadening the indication of CRT to patients with mild HF. The Multicenter Automatic Defibrillator Implantation Trial-Cardiac Resynchronization Therapy (MADIT-CRT), the Resynchronization-Defibrillation in Ambulatory Heart Failure Trial (RAFT) and Resynchronization Reverses Remodeling in Systolic Left Ventricular Dysfunction (REVERSE) trials enrolled patients primarily with mild HF, presenting with NYHA class I and II HF symptoms $(14,15)$. These studies have shown a significant reduction in HF events and improvement in clinical symptoms, as well as significant LV reverse remodeling. The subsequently published long-term follow-up of MADIT-CRT, and REVERSE studies confirmed sustained benefit of CRT in mild HF patients up to 5 years with reduction in HF events and a significant reduction in mortality $(16,17)$.

\section{Modifying Factors of Response to Cardiac Resynchronization Therapy}

Despite the overall beneficial effects of cardiac resynchronization therapy shown in numerous large clinical tri- als, nearly one third of the patients demonstrate a lack of echocardiographic or clinical response to CRT, they are so-called non-responders. Non-response has been the major focus of CRT research the past 25 years to optimize use, delivery, and care of CRT patients to optimize outcomes. Non-response to CRT is multifactorial, including baseline clinical charcteristics linked to unfavorable outcomes through CRT delivery, and post-implant factors, such as arrhythmias and CRT programming.

\section{Baseline Clinical Characteristics}

There have been a number of clinical factors associated with poor clinical response to CRT including diabetes, renal dysfunction, and ischemic cardiomyopathy (18). On the contrary, a superior response to CRT is predicted by female sex, non-ischemic etiology of cardiomyopathy, left bundle branch block, wide QRS, and a less dilated left ventricle (19). The amount of scar has been shown to negatively correlate with outcomes in patients with CRT and warrant further investigation. It is plausible that advanced scar formation might not be amenable to CRT and on the contrary, might be arrhythmogenic, especially if LV pacing occurs near a scar region as shown by previous studies. Important gender differences in CRT outcomes might be linked to the fact that women often present with non-ischemic cardiomyopathy and LBBB, a substrate that is the most responsive to biventricular pacing (20).

\section{QRS morphology and QRS duration}

Although CRT has been shown to be beneficial in HF patients with a low LVEF and a wide QRS, several early studies noted differences in response by QRS duration, and by the underlying ECG pattern at baseline, befo- 
TABLE 1. Randomized Past and *Ongoing Controlled Trials of Cardiac Resynchronization Therapy. Abbreviations: 6MWT, 6-min walk test; CARE-HF, Cardiac Resynchronization-Heart Failure; COMPANION, Comparison of Medical Therapy, Pacing and Defibrillation in Heart Failure; HF, heart failure; LV, left ventricular; LVEDD, left ventricular end-diastolic dimension; LVEF, left ventricular ejection fraction; LVESV, left ventricular end-systolic volume; MADITCRT, Multicenter Automatic Defibrillator Implantation TrialCardiac Resynchronization Therapy; MIRACLE, Multicenter InSync Randomized Clinical Evaluation; MIRACLE ICD, Multicenter InSync Implantable Cardioverter Defibrillator trial; MR, mitral regurgitation; MUSTIC, Multisite Simulation in Cardiomyopathies; NYHA, New York Heart Association; PATH-CHF, Pacing Therapies in Congestive Heart Failure trial; QOL, quality-of-life score; RAFT, Resynchronization-Defibrillation for Ambulatory Heart Failure; REVERSE, Resynchronization Reverses Remodeling in Systolic Left Ventricular Dysfunction; $\mathrm{VO}_{2}$, volume of oxygen

\begin{tabular}{|c|c|c|c|c|c|}
\hline Clinical Trial & Patients (n) & Primary end points & Secondary end points & LVEF (\%) & QRS (ms) \\
\hline MUSTIC-SR & 58 & 6MWT & $\begin{array}{l}\text { NYHA, QOL, Peak VO }{ }_{2}, \mathrm{MR}, \mathrm{LV} \text {, } \\
\text { Hosp, Mortality }\end{array}$ & $23 \pm 7$ & 174 \\
\hline MUSTIC-AF & 64 & 6MWT & $\begin{array}{l}\text { NYHA, QOL, Peak VO, } \mathrm{V}_{2} \text {, Hosp, } \\
\text { Mortality }\end{array}$ & $26 \pm 0$ & 206 \\
\hline PATH-CHF 2 & 41 & 6MWT, peak VO2 & $\begin{array}{l}\text { NHYA class, QOL, } \\
\text { Hospitalizations }\end{array}$ & $21 \pm 7$ & 175 \\
\hline $\begin{array}{l}\text { PATH-CHF-II: } \\
\text { (Europe) }\end{array}$ & 86 & 6MWT, peak VO2 & $\begin{array}{l}\text { NHYA class, QOL, } \\
\text { Hospitalizations }\end{array}$ & $21 \pm 7$ & 175 \\
\hline MIRACLE & 453 & 6MWT, NHYA, QOL & $\begin{array}{l}\text { Peak VO2, LVEF, LVEDD, MR, } \\
\text { Clin Response }\end{array}$ & $22 \pm 6$ & 166 \\
\hline COMPANION & 1520 & $\begin{array}{l}\text { All-cause mortality or } \\
\text { hospitalization }\end{array}$ & $\begin{array}{l}\text { All-cause mortality and cardiac } \\
\text { mortality }\end{array}$ & 21 & 159 \\
\hline CARE-HF & 814 & All-cause mortality & $\begin{array}{l}\text { NYHA, QOL, LVEF, LVESV, } \\
\text { Hospitalization for heart failure }\end{array}$ & 25 & 160 \\
\hline REVERSE & 610 & HF clinical composite score & LVESVi & $27 \pm 7$ & 153 \\
\hline MADIT-CRT & 1820 & HF or death & $\begin{array}{l}\text { LVESV, LVEDV change, multiple } \\
\text { HF events }\end{array}$ & $24 \pm 5$ & 162 \\
\hline RAFT & 1798 & $\begin{array}{l}\text { All-cause mortality or HF } \\
\text { hospitalization }\end{array}$ & $\begin{array}{l}\text { All-cause mortality, cardiac } \\
\text { mortality, HF hospitalization }\end{array}$ & $23 \pm 5$ & 158 \\
\hline RAFT AF* & 412 & HF hospitalization or death & Mortality, HF hosp, QoL, 6MWT, & n.a. & n.a. \\
\hline $\begin{array}{l}\text { BUDAPEST CRT } \\
\text { Upgrade* }^{*}\end{array}$ & 360 & $\begin{array}{l}\text { HF hospitalization, death, or } \\
\text { lack of LV remodeling }\end{array}$ & Mortality, HF hospitalization & n.a. & n.a. \\
\hline
\end{tabular}

re CRT implantation. The first large randomized trials designed to evaluate the effect of CRT on all-cause mortality, Cardiac Resynchronization-Heart Failure (CARE-HF), and Comparison of Medical Therapy, Pacing and Defibrillation in Heart Failure (COMPANION), enrolled only $6 \%$ and $29 \%$ of their patients with nonLBBB, respectively. The REVERSE study enrolled $38 \%$ of patients with non-LBBB, and MADIT-CRT enrolled $30 \%$ (21), allowing us with an opportunity to analyze response by QRS morphology.

Patients with a LBBB ECG pattern before device implantation have been suggested to derive a significant benefit from CRT-D, while those with non-LBBB ECG pattern were shown to derive less or no benefit, and our sub-study from MADIT-CRT suggested potential harm (22). Subsequent studies confirmed an association between QRS duration and outcomes in the REVERSE trial (23). A sub-analysis from the RAFT trial confirmed a link between QRS morphology, QRS duration and outcomes in LBBB, and no benefit in non-LBBB patients (24), similarly to a large U.S. nationwide registry, the National Cardiovascular Database Registry (NCDR) ICD Registry (25). Based on these observa- tions, CRT today is a Class I or Class Ila indication for CRT in symptomatic HF patients with LBBB $\geq 120 \mathrm{~ms}$ with a Class I indication for those with a QRS $\geq 150 \mathrm{~ms}$. For patients with non-LBBB, CRT is a Class Ila indication for a QRS duration $\geq 150 \mathrm{~ms}$, and a Class Ilb indication for a QRS duration of 120 to 149 ms (26).

The previously conducted and published ECHO-CRT (27) and RethinQ (28) studies assessed CRT indication for HF patients with a narrow QRS complex $<120 \mathrm{~ms}$ but failed to demonstrate a benefit. Therefore, CRT is currently not indicated for patients with a narrow QRS complex, unless they require frequent ventricular pacing $(>40 \%)$ to treat bradycardia, an indication tested in the BLOCK-HF study (29).

\section{Prolonged PR-interval}

A prolonged PR-interval may result in atrioventricular dyssynchrony, with altered transmitral left ventricular filling and possible serious adverse clinical consequences as discussed above (3), and it could potentialyly be another important determinant of CRT response. We have previously shown in a sub-study of the MADIT-CRT trial that HF patients with non-LBBB ECG 
pattern and a prolonged PR-interval $\geq 230 \mathrm{~ms}$ derive a significant clinical benefit from CRT-D as compared to ICD (30), with a $73 \%$ risk reduction in HF or death, and an $81 \%$ risk reduction in all-cause mortality. NonLBBB patients with a normal PR-interval $<230$ ms were however exposed to a harm from CRT-D with a more than two-fold increase in mortality when compared to ICD-only (interaction p-value<0.001) (30). We subsequently confirmed in the MADIT-CRT long-term follow-up sub-study suggesting a sustained clinical benefit during a median follow-up of 5.6 years (31). A prior study showed similar associations from COMPANION with a prolonged PR-interval in more advanced HF patients (32), and in those with a narrow QRS enrolled in RethinQ (33). However, more recent studies challenged these findings using data from the NCDR ICD Registry, however, this cohort lacked randomization (34). Further studies are needed in this cohort.

\section{Controversies in CRT and Future Directions}

\section{Frequent Right Ventricular Pacing and CRT}

The underlying concept for the benefit of physiologic, AV-sequential pacing in $\mathrm{HF}$ patients with a prolonged PR-interval is well known. Previously reported case series on right ventricular (RV) DDD pacing with shorter AV-delay in HF patients and low ejection fraction in the 1990's reported an improvement in HF symptoms (35). It has been previously shown that frequent apical right ventricular pacing has deleterious effects $(36,37)$, especially in patients with a depressed left ventricular duntion. In this population, upgrading the pacing or ICD device to CRT could potentially improve outcomes. There have been a few retrospective studies evaluating the effects of CRT upgrade in patients with frequent RV apical pacing showing improvements in reverse remodeling, and functional capacity, however, none of these studies were randomized or had an appropriate control group (38). A large, randomized controlled clinical trial, the BUDAPEST CRT Upgrade study is currently ongoing to prospectively evaluate the effects of CRT upgrade from conventional PM or ICD therapy in patients with intermittent or permanent right ventricular (RV) septal/apical pacing, reduced LVEF, and symptomatic HF. This prospective, randomized, multicentre, investigator-sponsored clinical trial will enroll a total of 360 subjects with LVEF $\leq 35 \%$, NYHA functional class II-IVa, paced QRS $\geq 150 \mathrm{~ms}$, and RV pacing $\geq 20 \%$. Patients will be randomized to CRT-D vs. ICD in a $3: 2$ ratio and they will be followed for 12 months. The primary composite endpoint is all-cause mortality, or a HF event, or less than $15 \%$ reduction in LV end-systolic volume at 12 months (Table 2). Secondary endpoints are all-cause mortality, all-cause mortality or HF event, and LV volume reduction at 12 months (https://clinicaltrials. gov/ct2/show/NCT02270840).
Use of CRT-Defibrillator vs. CRT-Pacemaker It has been a long debate whether CRT with a defibrillator (CRT-D) is superior to CRT with a pacemaker (CRT-P) and whom should be implanted with a CRT-D vs. a CRT-P device. There have been no studies directly comparing the benefit of CRT-D to CRT-P in unselected patients in a randomized fashion. Patients with nonischemic cardiomyopathy, those with LBBB, women, are at a significantly lower risk of ventricular arrhythmias and derive significant LV reverse remodeling from CRT, all negating the potential need and benefit of an added defibrillator (39). In addition, defibrillator leads are associated with a higher risk of complications, lead fractures, and infections. Currently, CRT-P use is common in Europe due to the financial constraints of many countries (40). Our very own, single-center, high volume registry data additionally suggested that selected non-ischemic patients who were more often women, and older, did not have an improved survival with CRT-D as compared to CRT-P (41). Further studies are currently underway to prospectively evaluate outcomes of CRT-D vs. CRT-P in a randomized fashion in both ischemic and non-ischemic patients.

\section{Left Ventricular Lead Location and CRT Programming}

Several previous studies highlighted the importance of LV lead location for CRT outcomes. Early studies suggested that lateral or posterolateral LV lead location is associated with better outcomes while studies from MADIT-CRT and REVERSE highlighted the importance of avoiding apical LV lead locations to reduce the risk of HF or death (42). A subsequent analysis also suggested that anterior LV lead placement is linked to an increased risk of ventricular arrhythmias and should be avoided (43). Several attempts have been made to individually optimize LV lead placement and target the latest activated area identified by imaging studies (44, 45) however, despite initial promising findings none of these techniques are currently employed in routine clinical practice. A recent study focusing on CRT non-responder non-LBBB patients to optimize lead placement using LV electrical delay measured by Q-LV, also failed to meet its primary end point (https://clinicaltrials.gov/ ct2/show/NCT01983293). Newer technical advancements, such as quadripolar LV leads with multipoint pacing, and individually optimized pacing sequences are currently studied to further improve outcomes of CRT non-responders.

Optimal CRT programming is a cornerstone of beneficial CRT outcomes, with several studies suggesting that the higher the biventricular pacing percentage is, the better the outcomes. Ruwald et al. (46) showed that $>97 \%$ of biventricular pacing was linked with improved survival in MADIT-CRT. Prior studies assessing optimal CRT programming and outcomes using echocardiography optimization vs. "out of the box" device settings 
vs. dynamic optimization techniques have been however unsuccessful in de novo CRT recipients (47-49), but showed some benefits in CRT non-responders (50).

\section{Atrial Fibrillation}

HF patients with atrial fibrillation have been shown to have adverse outcomes with an implanted CRT. In CRT recipients, lack of biventricular pacing and abrogation of the remodeling process are of particular concern. Many randomized clinical trials also excluded patients with persistent/permanent atrial fibrillation, significantly limiting our understanding of CRT outcomes in this cohort. A currently ongoing randomized clinical trial, RAFT-AF (https://clinicaltrials.gov/ct2/show/NCT01420393) is evaluating the role of catheter ablation with PV antral isolation and LA substrate ablation vs. rate control in CRT recipients, hopefully shedding more lights on treatment outcomes in this difficult to treat patient population.

Another prospective, randomized clinical trial of 80 patients, JAVA-CRT is assessing the role of AV-junctional ablation in CRT patients with high burden of atrial fibrillation to improve outcomes (https://clinicaltrials.gov/ct2/ show/NCT02946853).

\section{Conclusions}

Cardiac resynchronization therapy has evolved as a mainstream therapy for heart failure in patients with mild to advanced heart failure symptoms, severely depressed left ventricular ejection fraction, and a wide QRS. Short- and long-term outcomes have been favorable, nevertheless influences by various clinical characteristics, and comorbidities. Tailored LV lead implantation or CRT programming does not further improve outcomes however further studies are currently underway. The utility of CRT upgrade in patients with chronic RV apical/septal pacing, rhythm control/AV junctional ablation in patients with atrial fibrillation, and the appropriate use of CRT-D vs. CRT-P are currently unresolved issues that need further investigation to optimize outcomes.

\section{References}

1. Ghio S, Constantin C, Klersy C, et al. Interventricular and intraventricular dyssynchrony are common in heart failure patients, regardless of QRS duration. European heart journal 2004; 25: 571-8. DOI: 10.1016/j ehj.2003.09.030

2. Fantoni C, Kawabata M, Massaro R, et al. Right and left ventricular activation sequence in patients with heart failure and right bundle branch block: a detailed analysis using three-dimensional non-fluoroscopic electroanatomic mapping system. Journal of cardiovascular electrophysiology 2005; 16: 112-9: discussion 120-1. DOI: 10.1046/j.15408167.2005.40777.x

3. Salden F, Kutyifa V, Stockburger M, Prinzen FW, Vernooy K. Atrioventricular dromotropathy: evidence for a distinctive entity in heart failure with prolonged PR interval? Europace : European pacing, arrhythmias, and cardiac electrophysiology : journal of the working groups on cardiac pacing, arrhythmias, and cardiac cellular electrophysiology of the European Society of Cardiology 2018; 20: 1067-1077. DOI: 10.1093/europace/eux207

4. Breithardt $O A$, Sinha AM, Schwammenthal E, et al. Acute effects of cardiac resynchronization therapy on functional mitral regurgitation in advanced systolic heart failure. Journal of the American College of Cardiology 2003; 41: 765-70. DOI: 10.1016/S0735-1097(02)02937-6 5. Auricchio A, Stellbrink C, Sack S, et al. Long-term clinical effect of hemodynamically optimized cardiac resynchronization therapy in patients with heart failure and ventricular conduction delay. Journal of the American College of Cardiology 2002; 39: 2026-33. DOI: 10.1016/ S0735-1097(02)01895-8

6. Cazeau S, Leclercq C, Lavergne T, et al. Effects of multisite biventricular pacing in patients with heart failure and intraventricular conduction delay. The New England journal of medicine 2001; 344: 873-80. DOI: 10.1056/NEJM200103223441202

7. Solomon SD, Foster E, Bourgoun M, et al. Effect of cardiac resynchronization therapy on reverse remodeling and relation to outcome: multicenter automatic defibrillator implantation trial: cardiac resynchronization therapy. Circulation 2010; 122: 985-92. DOI:10.1161/CIRCULATIONAHA.110.955039

8 Young JB, Abraham WT, Smith AL, et al. Combined cardiac resynchronization and implantable cardioversion defibrillation in advanced chronic heart failure: the MIRACLE ICD Trial. JAMA: the journal of the American Medical Association 2003; 289: 2685-94. DOI:10.1001/ jama.289.20.2685

9. Bristow MR, Saxon LA, Boehmer J, et al. Cardiac-resynchronization therapy with or without an implantable defibrillator in advanced chronic heart failure. The New England journal of medicine 2004; 350: 2140 50. DOI: 10.1056/NEJMoa032423

10. Cleland JG, Daubert JC, Erdmann E, et al. The effect of cardiac resynchronization on morbidity and mortality in heart failure. The New England journal of medicine 2005; 352: 1539-49. DOI: 10.1056/NEJMoa050496

11. Brignole M, Auricchio A, Baron-Esquivias G, et al. 2013 ESC Guidelines on cardiac pacing and cardiac resynchronization therapy: the Task Force on cardiac pacing and resynchronization therapy of the European Society of Cardiology (ESC). Developed in collaboration with the European Heart Rhythm Association (EHRA). European heart journal 2013; 34: 2281-329. DOI:10.1093/europace/eut206

12. Dickstein K, Normand C, Auricchio A, et al. CRT Survey II: a European Society of Cardiology survey of cardiac resynchronisation therapy in 11088 patients-who is doing what to whom and how? European journal of heart failure 2018; 20: 1039-1051. DOI: 10.1002/ejhf.1142

13. Rivero-Ayerza M, Theuns DA, Garcia-Garcia HM, Boersma E, Simoons M, Jordaens LJ. Effects of cardiac resynchronization therapy on overall mortality and mode of death: a meta-analysis of randomized controlled trials. European heart journal 2006; 27: 2682-8. DOI:10.1093/eurheartj/ehl203

14. Tang AS, Wells GA, Talajic M, et al. Cardiac-resynchronization therapy for mild-to-moderate heart failure. The New England journal of medicine 2010; 363: 2385-95. DOI:10.1056/NEJMoa1009540

15. Linde C, Abraham WT, Gold MR, St John Sutton M, Ghio S, Daubert $\mathrm{C}$. Randomized trial of cardiac resynchronization in mildly symptomatic heart failure patients and in asymptomatic patients with left ventricular dysfunction and previous heart failure symptoms. Journal of the American College of Cardiology 2008; 52: 1834-43. DOI: 10.1016/j. jacc. 2008.08.027

16. Goldenberg I, Kutyifa V, Moss AJ. Survival with cardiac-resynchronization therapy. The New England journal of medicine 2014; 371: 4778. DOI: 10.1056/NEJMoa1401426

17. Linde C, Gold MR, Abraham WT, et al. Long-term impact of cardiac resynchronization therapy in mild heart failure: 5 -year results from the REsynchronization reVErses Remodeling in Systolic left vEntricular dysfunction (REVERSE) study. European heart journal 2013; 34: 2592-9. DOI: 10.1093/eurheartj/eht160

18. Naqvi SY, Jawaid A, Goldenberg I, Kutyifa V. Non-response to Cardiac Resynchronization Therapy. Current heart failure reports 2018; 15: 315-321. DOI: 10.1007/s11897-018-0407-7

19. Goldenberg I, Moss AJ, Hall WJ, et al. Predictors of response to cardiac resynchronization therapy in the Multicenter Automatic Defibrillator Implantation Trial with Cardiac Resynchronization Therapy (MADIT-CRT). Circulation 2011; 124: 1527-36. DOI:10.1161/CIRCULATIONAHA.110.014324 
20. Arshad A, Moss AJ, Foster E, et al. Cardiac resynchronization therapy is more effective in women than in men: the MADIT-CRT (Multicenter Automatic Defibrillator Implantation Trial with Cardiac Resynchronization Therapy) trial. Journal of the American College of Cardiology 2011; 57: 813-20. DOI: 10.1016/j.jacc.2010.06.061

21. Moss AJ, Hall WJ, Cannom DS, et al. Cardiac-resynchronization therapy for the prevention of heart-failure events. The New Eng land journal of medicine 2009; 361: 1329-38. DOI:10.1056/NEJMoa0906431

22. Zareba W, Klein H, Cygankiewicz I, et al. Effectiveness of Cardiac Resynchronization Therapy by QRS Morphology in the Multicenter Automatic Defibrillator Implantation Trial-Cardiac Resynchronization Therapy (MADIT-CRT). Circulation 2011; 123: 1061-72. DOI:10.1161/ CIRCULATIONAHA.110.960898

23. Gold MR, Thebault C, Linde C, et al. Effect of QRS duration and morphology on cardiac resynchronization therapy outcomes in mild heart failure: results from the Resynchronization Reverses Remodeling in Systolic Left Ventricular Dysfunction (REVERSE) study. Circulation 2012; 126: 822-9. DOI: 10.1161/CIRCULATIONAHA.112.097709 24. Birnie DH, Ha A, Higginson L, et al. Impact of QRS morphology and duration on outcomes after cardiac resynchronization therapy: Results from the Resynchronization-Defibrillation for Ambulatory Heart Failure Trial (RAFT). Circulation Heart failure 2013; 6: 1190-8. DOI:10.1161/ CIRCHEARTFAILURE.113.000380

25. Peterson PN, Greiner MA, Qualls LG, et al. QRS duration, bundle-branch block morphology, and outcomes among older patients with heart failure receiving cardiac resynchronization therapy. Jama 2013; 310: 617-26. DOI: 10.1001/jama.2013.8641

26. Ponikowski P, Voors AA, Anker SD, et al. ESC Scientific Document Group. 2016 ESC Guidelines for the diagnosis and treatment of acute and chronic heart failure: The Task Force for the diagnosis and treatment of acute and chronic heart failure of the European Society of Cardiology (ESC) Developed with the special contribution of the Heart Failure Association (HFA) of the ESC. European Heart Journal, Volume 37, Issue 27, 14 July 2016, Pages 2129-2200, https://doi.org/10.1093/eurheartj/ehw128 27. Ruschitzka F, Abraham WT, Singh JP, et al. Cardiac-resynchronization therapy in heart failure with a narrow QRS complex. The New England journal of medicine 2013; 369: 1395-405. DOI:10.1056/NEJMoa1306687

28. Beshai JF, Grimm RA, Nagueh SF, et al. Cardiac-resynchronization therapy in heart failure with narrow QRS complexes. The New England journal of medicine 2007; 357: 2461-71. DOI:10.1056/NEJMoa0706695

29. Curtis AB, Worley SJ, Adamson PB, et al. Biventricular pacing for atrioventricular block and systolic dysfunction. The New England journal of medicine 2013; 368: 1585-93. DOI:10.1056/NEJMoa1210356

30. Kutyifa V, Stockburger M, Daubert JP, et al. PR interval identifies clinical response in patients with non-left bundle branch block: a Multicenter Automatic Defibrillator Implantation Trial-Cardiac Resynchronization Therapy sub-study. Circulation Arrhythmia and electrophysiology 2014; 7: 645-51. DOI:10.1161/CIRCEP.113.001299

31. Stockburger M, Moss AJ, Klein HU, et al. Sustained clinical benefit of cardiac resynchronization therapy in non-LBBB patients with prolonged PR-interval: MADIT-CRT long-term follow-up. Clinical research in cardiology: official journal of the German Cardiac Society 2016; 105 : 944-952. DOI:10.1007/s00392-016-1003-Z

32. Olshansky B, Day JD, Sullivan RM, Yong P, Galle E, Steinberg JS Does cardiac resynchronization therapy provide unrecognized benefit in patients with prolonged PR intervals? The impact of restoring atrioventricular synchrony: an analysis from the COMPANION Trial. Heart rhythm: the official journal of the Heart Rhythm Society 2012; 9: 34-9. DOI:10.1016/j.hrthm.2011.07.038

33. Joshi NP, Stopper MM, Li J, Beshai JF, Pavri BB. Impact of baseline $\mathrm{PR}$ interval on cardiac resynchronization therapy outcomes in patients with narrow QRS complexes: an analysis of the ReThinQ Trial. Journal of interventional cardiac electrophysiology: an international journal of arrhythmias and pacing 2015; 43: 145-9. DOI:10.1007/s10840-015-9999-y 34. Friedman DJ, Bao H, Spatz ES, Curtis JP, Daubert JP, Al-Khatib SM. Association Between a Prolonged PR Interval and Outcomes of Cardiac Resynchronization Therapy: A Report From the National Cardiovascular Data Registry. Circulation 2016; 134: 1617-1628. DOI: 10.1161/CIRCULATIONAHA.116.022913

35. Hochleitner M, Hortnagl H, Ng CK, Gschnitzer F, Zechmann W. Usefulness of physiologic dual-chamber pacing in drug-resistant idio- pathic dilated cardiomyopathy. The American journal of cardiology 1990; 66: 198-202.

36. Kutalek SP, Sharma AD, McWilliams MJ, et al. Effect of pacing for soft indications on mortality and heart failure in the dual chamber and VVI implantable defibrillator (DAVID) trial. Pacing and clinical electrophysiology: PACE 2008; 31: 828-37. DOI:10.1111/j.15408159.2008.01106.x

37. Lamas GA, Lee KL, Sweeney MO, et al. Ventricular pacing or dual-chamber pacing for sinus-node dysfunction. The New England journal of medicine 2002; 346: 1854-62. DOI:10.1056/NEJMoa013040 38. Kosztin A, Vamos M, Aradi D, et al. De novo implantation vs. upgrade cardiac resynchronization therapy: a systematic review and meta-analysis. Heart failure reviews 2018; 23: 15-26. DOI:10.1007/ s10741-017-9652-1

39. Barra S, Providencia R, Boveda S, et al. Do women benefit equally as men from the primary prevention implantable cardioverter-defibrillator? Europace: European pacing, arrhythmias, and cardiac electrophysiology: journal of the working groups on cardiac pacing, arrhythmias, and cardiac cellular electrophysiology of the European Society of Cardiology 2017. DOI:10.1093/europace/eux203

40. Merkely B, Roka A, Kutyifa V, et al. Tracing the European course of cardiac resynchronization therapy from 2006 to 2008. Europace: European pacing, arrhythmias, and cardiac electrophysiology: journal of the working groups on cardiac pacing, arrhythmias, and cardiac cellular electrophysiology of the European Society of Cardiology 2010; 12 : 692-701. DOl:10.1093/europace/euq041

41. Kutyifa V, Geller L, Bogyi P, et al. Effect of cardiac resynchronization therapy with implantable cardioverter defibrillator versus cardiac resynchronization therapy with pacemaker on mortality in heart failure patients: results of a high-volume, single-centre experience. European journal of heart failure 2014; 16: 1323-30. DOI:10.1002/ejhf.185

42. Singh JP, Klein HU, Huang DT, et al. Left ventricular lead position and clinical outcome in the multicenter automatic defibrillator implantation trial-cardiac resynchronization therapy (MADIT-CRT) trial. Circulation 2011; 123: 1159-66. DOI:10.1161/CIRCULATIONAHA.110.000646 43. Kutyifa V, Zareba W, McNitt $S$, et al. Left ventricular lead location and the risk of ventricular arrhythmias in the MADIT-CRT trial. European heart journal 2013; 34: 184-90. DOI:10.1093/eurheartj/ehs334

44. Khan FZ, Virdee MS, Palmer CR, et al. Targeted Left Ventricular Lead Placement to Guide Cardiac Resynchronization Therapy: The TARGET Study: A Randomized, Controlled Trial. Journal of the American College of Cardiology 2012. DOI:10.1016/j.jacc.2011.12.030 45. Barra S, Yu E, Khan F, Dutka D, Virdee M. An individualized left ventricular lead-targeting strategy improves long-term survival of cardiac resynchronization therapy patients and influences the benefit of the implantable cardioverter-defibrillator: a long-term follow-up of the TARGET study. Journal of cardiovascular medicine (Hagerstown, Md) 2017; 18: 553-555. DOI:10.2459/JCM.0000000000000396

46. Ruwald AC, Kutyifa $\mathrm{V}$, Ruwald MH, et al. The association between biventricular pacing and cardiac resynchronization therapy-defibrillator efficacy when compared with implantable cardioverter defibrillator on outcomes and reverse remodelling. European heart journal 2014. DOI:10.1093/eurheartj/ehu294

47. Stein KM, Ellenbogen KA, Gold MR, et al. SmartDelay determined AV optimization: a comparison of AV delay methods used in cardiac resynchronization therapy (SMART-AV): rationale and design Pacing and clinical electrophysiology: PACE 2010; 33: 54-63. DOI: 10.1111/j.15408159.2009.02581.x

48. Ellenbogen KA, Gold MR, Meyer TE, et al. Primary results from the SmartDelay determined AV optimization: a comparison to other AV delay methods used in cardiac resynchronization therapy (SMART-AV) trial: a randomized trial comparing empirical, echocardiography-guided, and algorithmic atrioventricular delay programming in cardiac resynchronization therapy. Circulation 2010; 122: 2660-8. DOI:10.1161/CIRCULATIONAHA.110.992552

49. Kedia N, Ng K, Apperson-Hansen C, et al. Usefulness of atrioventricular delay optimization using Doppler assessment of mitral inflow in patients undergoing cardiac resynchronization therapy. The American journal of cardiology 2006; 98: 780-5. DOI:10.1016/j.amjcard.2006.04.017

50. Mullens W, Grimm RA, Verga T, et al. Insights from a cardiac resynchronization optimization clinic as part of a heart failure disease management program. Journal of the American College of Cardiology 2009; 53: 765-73 DOI:10.1016/j.jacc.2008.11.024 